\title{
Ferramenta de Apoio ao ensino de Física utilizando Realidade Aumentada
}

Title: Tool to Aid Teaching Physics through Augmented Reality

\author{
Márcio Augusto Carvalho Viegas \\ Departamento de Ciência da \\ Computação (DCC/ICE) \\ Universidade Federal de Juiz de Fora \\ marcio_augusto89@hotmail.com
}

\author{
Marcelo Bernardes Vieira \\ Departamento de Ciência da \\ Computação (DCC/ICE) \\ Universidade Federal de Juiz de Fora \\ marcelo.bernardes@ufjf.edu.br
}

Rodrigo Luis de Souza da Silva Departamento de Ciência da Computação (DCC/ICE)

Universidade Federal de Juiz de Fora rodrigoluis@ice.ufjf.com

\begin{abstract}
Resumo Este artigo apresenta uma ferramenta de apoio ao ensino de física utilizando Realidade Aumentada. Os principais conceitos sobre Realidade Aumentada e os frameworks da área serão apresentados. As funções que simulam os eventos físicos serão explicadas e desenvolvidas. Neste trabalho nenhuma biblioteca clássica de simulação física foi utilizada. A ferramenta foi dividida em vários módulos da física e, para cada um destes módulos, um sistema baseado em Realidade Aumentada foi proposto. A proposta central deste trabalho é mostrar que ferramentas baseadas em Realidade Aumentada podem potencialmente tornar o estudo de física mais lúdico e interessante.
\end{abstract}

Palavras-Chave: Realidade Aumentada, Computação Gráfica, Ensino.

\begin{abstract}
This paper presents a tool to aid teaching physics through Augmented Reality. The main concepts regarding Augmented Reality and the frameworks of this field will be presented. The functions that simulates the physical events will be explained and developed. In this work no classic physics library was used. The tool was divided in many physical modules and, for each one of them, a system based on Augmented Reality was proposed. The main proposal of this work is to show that tools based on Augmented Reality can potentially make the study of physics more playful and interesting.
\end{abstract}

Keywords: Augmented Reality, Computer Graphics, Teaching. 


\section{Introdução}

Recentemente, a vasta utilização de recursos tecnológicos em instituições de ensino gerou um crescente interesse no desenvolvimento de ferramentas que auxiliem o aprendizado. Em decorrência disso, formou-se um extenso campo multidisciplinar na área da ciência da computação focado na pesquisa e desenvolvimento dessas ferramentas.

O interesse em desenvolver uma ferramenta de ensino de física utilizando Realidade Aumentada (RA) é proveniente das vantagens que essa tecnologia pode proporcionar aos estudantes. A RA permite que o usuário controle o sistema, aguça a sua percepção através da visão e possibilita que os objetos virtuais reproduzam as leis da física [15].

Muitos alunos de ensino fundamental e médio possuem dificuldade em aprender física. Enumera-se dois principais problemas causadores dessa realidade. Primeiramente, falta uma forma para representar o modelo físico em três dimensões, tal como eles acontecem. Não menos importante, simulações de eventos físicos podem acontecer de forma não trivial. Assim, torna-se um problema a ausência de meios que possibilitem ao usuário interagir com essas simulações e observar as consequências de possíveis modificações em um modelo físico.

O uso de recursos tecnológicos, como a RA, associados a um treinamento e uma conscientização profissional dos educadores, mostra-se como possível alternativa à difícil tarefa de ensinar física.

Os sistemas físicos estudados, tanto a nível de ensino médio como de superior, simulam situações reais que ocorrem em nosso universo. Diferentemente do modelo atual que utiliza livros, a RA permite a visualização do modelo físico em três dimensões. É nesse contexto que a RA torna as explicações mais intuitivas, pois permite a visualização dos eventos físicos na forma como eles acontecem.

Um estudante poderá modificar as informações veiculadas ao sistema físico, recebendo em tempo real os resultados que suas ações causaram na simulação do evento físico. Essa possibilidade aumenta a interação do aluno com a matéria e consequentemente o interesse do mesmo por ela.

O objetivo principal deste trabalho é a construção de uma ferramenta de apoio ao ensino de física utilizando RA. Sistemas serão simulados e exibidos utilizando-se a RA. Professores serão capazes, com a ajuda da ferramenta desenvolvida nesse trabalho, de explicitar melhor os eventos físicos que pretendem ensinar à sua classe.

Pretende-se que a ferramenta seja suficientemente in- tuitiva para atrair o interesse dos professores, e de fácil manuseio para impedir que profissionais sem muito conhecimento técnico desistam de utilizá-la. Dessa forma, será implementado uma forma intuitiva de interação entre o usuário e a aplicação. Objetos reais permitirão que o usuário modifique o modelo físico. $\mathrm{O}$ usuário poderá controlar as variáveis do sistema físico, como o peso dos objetos e a velocidade inicial de cada um deles, utilizando marcadores de papel.

\section{Trabalhos Relacionados}

Propõe-se em [6] uma aplicação em RA para o ensino de física mecânica. Os alunos são habilitados a construir ativamente os seus próprios experimentos e estudá-los em um mundo tridimensional virtual. Uma variedade de ferramentas são fornecidas para analisar as forças, massa, caminhos e outras propriedades de objetos antes, durante e após os experimentos. A aplicação em questão, diferese do trabalho aqui proposto, devido aos recursos tecnológicos de alto custo, como tablets e HMD (Head Mounted Display), necessários para utilizá-la.

Baseado no sistema colaborativo de RA Studierstube, os autores em [5] propõem uma ferramenta de RA para o ensino de física e matemática. Como forma de suportar as diferentes formas de interação entre professor e alunos, foram implementados métodos flexíveis que se adaptam a diversos contextos. Entretanto, não há formas de se alterar as variáveis do modelo virtual apresentado e inserido no mundo real.

Uma interface interativa multimídia de RA para $E$ Learning é apresentada em [9]. São utilizados áudio e vídeo para ensinar o conceito de máquina de estados finitos, assunto comumente lecionado nos cursos de ciência da computação. Utiliza-se o mouse para estabelecer uma interação entre o usuário e o sistema virtual.

Em [4] são apresentadas as vantagens da utilização da RA no ensino de química, mais precisamente no ensino de arranjos moleculares de três dimensões. Um cubo, com marcadores distintos em cada uma de suas faces, é utilizado para rotacionar a estrutura química possibilitando ao estudante analisar a mesma em todos os ângulos e em três dimensões. Assemelha-se à ferramenta aqui proposta, a ideia de utilizar os próprios marcadores, objetos reais, para modificar os objetos virtuais.

É conveniente ressaltar que a utilização da Realidade Aumenta para o ensino não está limitada as áreas de tecnologia e exatas. O trabalho desenvolvido em [12] apresenta a extensão de um simulador de partos para treinamento médico com um sistema de RA. O uso da ferramenta proposta propicia um aumento na eficiência do 
treinamento, já que o médico se concentra mais no modelo real do que na tela do computador remoto. Nesse tipo de protótipo existe a dificuldade em rastrear a perfeita localização do padrão estipulado onde serão inseridos os objetos virtuais.

\section{Realidade Aumentada}

O termo Realidade Aumentada pode ser definido de várias maneiras. Uma mistura do mundo real com o virtual; um sistema que possibilita a existência de objetos virtuais no mundo real; e uma particularização de realidade misturada, com a predominância do mundo real [7].

Pode-se imaginar a RA como uma variação da Realidade Virtual (RV). A princípio, a RA e a RV diferem-se no grau de realidade que ambos oferecem em suas aplicações. Na RV o usuário é imerso em um mundo virtual e fica impossibilitado de assimilar o mundo real a sua volta. Já a RA permite ao usuário ver o mundo real em conjunto com os objetos virtuais sobrepostos ao mesmo [2].

Basicamente, a RA possibilita inserir objetos virtuais no mundo real e permite que objetos reais manipulem o cenário virtual. Na Figura 1 há um carro e um vaso, ambos virtuais, sobre uma mesa real.

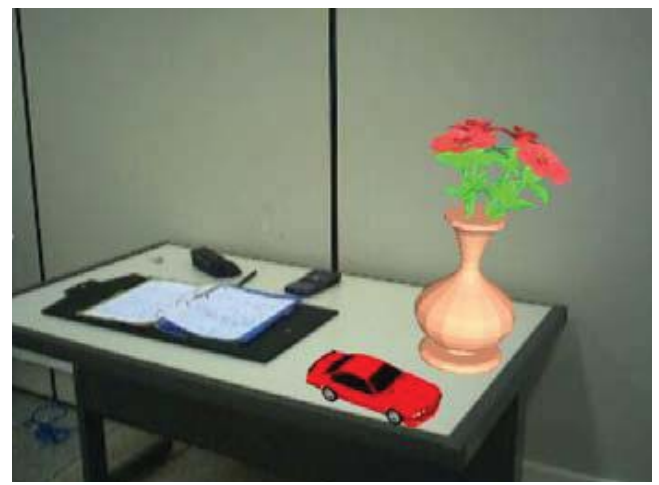

Figura 1: Exemplo de sistema de RA. Neste exemplo vaso e carro são virtuais [7].

Os sistemas de RA podem ser classificados conforme o tipo de display disponível [3]. Apesar das diferenças que esses sistemas possuem, ambos aguçam as habilidades visuais e espaciais de seus usuários e constituem um novo e promissor paradigma de interface entre usuário e computador [10].

\subsection{Sistemas de visão por vídeo baseado em monitor}

O conjunto webcam e monitor, devido ao custo baixo, é o mais utilizado nas aplicações de RA. A webcam é responsável por capturar a cena real. Essa cena, após capturada, é misturada com os objetos virtuais gerados pelo computador (Figura 2). Por fim, o mesmo exibe o resultado (mundo real mais objetos virtuais) em um monitor.

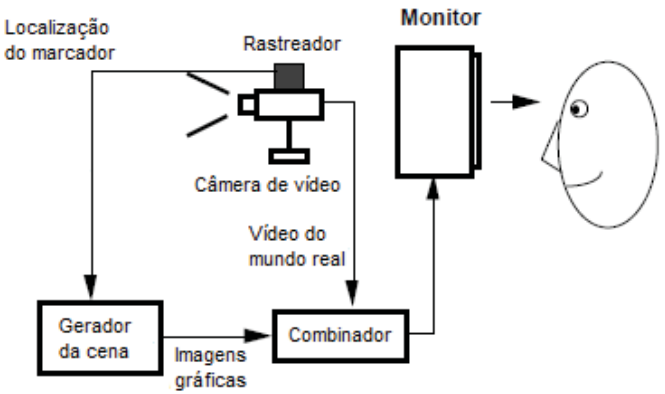

Figura 2: Funcionamento de um sistema de visão por vídeo baseado em monitor. Adaptado de [1].

Apesar de ser mais barato e de fácil ajuste, em sistemas de visão por vídeo baseado em monitor o ponto de vista do usuário é fixo. Ainda, aplicações tridimensionais são afetadas, já que a imagem no monitor é feita em duas dimensões [5].

\subsection{Sistemas de visão ótica direta}

Em sistemas de visão ótica direta (Figura 3) o usuário tem liberdade para movimentar-se pois o ponto de visão não é fixo. Consequentemente, a sensação de que os objetos virtuais fazem parte do mundo real é ainda maior.

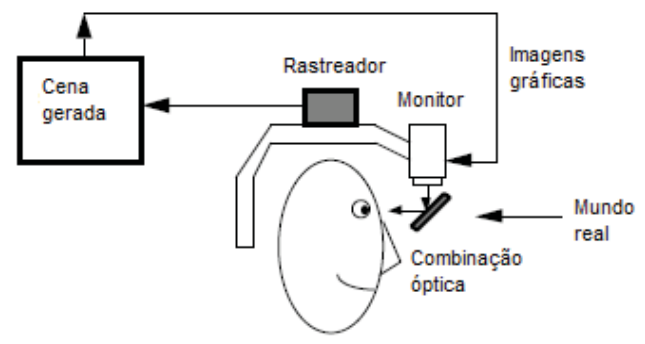

Figura 3: Funcionamento de um sistema de visão ótica direta adaptado de [1].

Devido a necessidade das superfícies de projeção, esse sistema fica restrito às condições do espaço real [5]. Além disso, se comparado ao sistema apresentado anteriormente, os equipamentos necessários para utilizar esse sistema possuem custos elevados.

\subsection{Framework utilizado}

O ARToolKit é um software livre bastante utilizado no desenvolvimento de aplicações de RA. A função dele é definir a localização onde serão impressos os objetos 
virtuais na cena real. Para isso, o ARToolkit utiliza matrizes de transformação e marcadores [1].

Marcadores são objetos reais que obedecem um padrão em sua construção e servem para posicionar os objetos virtuais na cena real (Figura 4). Os marcadores do ARToolKit, seguem o seguinte padrão: devem ter uma borda contínua (branca ou preta) sobre um fundo contrastante; cada borda precisa ter a espessura de vinte e cinco por cento do comprimento do marcador; precisa ser necessariamente um quadrado; e a imagem do centro, que distingue um marcador de outro, não deve ser rotacionalmente simétrica.

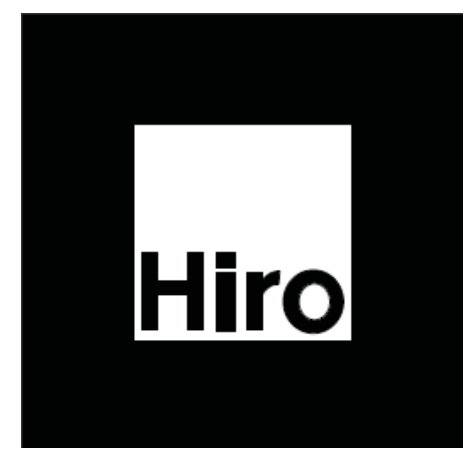

Figura 4: Marcador utilizado pelo ARToolKit.

Quando um marcador é reconhecido, o ARToolKit calcula a transformação da câmera relativa a esse marcador para que o objeto virtual possa ser desenhado. Assim, a localização de um objeto virtual estará sempre em função de um marcador ou de vários.

O SudaRA (Suporte ao Desenvolvimento de Aplicações em RA) é um framework de suporte ao desenvolvimento de aplicações de RA desenvolvido em $\mathrm{C}++$ e baseado no ARToolKit [13].

O framework oferece recursos como suporte a modelos 3D, som, rede e monitoramento de múltiplos marcadores. Além disso, o SudaRA destaca-se em relação ao ARToolKit pelo fato de facilitar o trabalho do desenvolvedor propiciando uma interface simples e bem estruturada.

Os dois frameworks citados foram testados nesta aplicação, mas optou-se pelo uso do SudaRA.

\section{Modelo físico}

Neste capítulo serão apresentados os principais conceitos da física mecânica e os modelos físicos dos sistemas propostos que serão implementados. Primeiramente, os termos da física mecânica serão discutidos. Esses serão a base para resolver as incógnitas dos eventos físicos que serão simulados. Por fim, avalia-se esses eventos físicos e descreve-se as fórmulas necessárias para a implementação de cada sistema.

\subsection{Movimento uniforme}

Define-se movimento uniforme como sendo aquele movimento que tem velocidade constante em qualquer instante ou intervalo de tempo. No movimento uniforme a variação da velocidade é igual a zero, pois a velocidade final é igual à velocidade inicial. Conclui-se que, para este movimento, a aceleração é sempre constante e igual a zero.

Sendo $\Delta \mathrm{S}$ a variação da posição do móvel e $\Delta \mathrm{t}$ a variação do tempo, pode-se expressar a velocidade como:

$v=\frac{\Delta \mathrm{S}}{\Delta \mathrm{t}}$

Considerando o tempo inicial igual a zero na Equação 1, temos a função horária do movimento uniforme (Equação 2).

$S=S_{0}+v t$

Essa é uma função de primeiro grau e é chamada de função horária da posição. Através dela podemos determinar a posição de um móvel num determinado instante.

\subsection{Movimento uniformemente variado}

O movimento uniformemente variado é o movimento no qual a velocidade varia uniformemente no decorrer do tempo. O movimento caracteriza-se por haver uma aceleração diferente de zero e constante.

A aceleração instantânea do móvel $a_{m}$ é dada pela Equação 3:

$a_{m}=\frac{\Delta v}{\Delta \mathrm{t}}$

Sabe-se que $\Delta v$ é a variação da velocidade do móvel e $\Delta \mathrm{t}$ é a variação do tempo. Assim, supondo $t_{0}=0$, da Equação 3 tem-se que a velocidade de um móvel que possui movimento uniformemente variado em um instante de tempo igual a $t$ é dada pela Equação 4:

$v=v_{0}+a t$

Essa é a função horária da velocidade do movimento uniformemente variado. Ela descreve a velocidade em função do tempo.

\subsection{Energia cinética}

Caracteriza-se Energia cinética $\left(E_{c}\right)$ como sendo a quantidade de trabalho a ser realizado sobre um objeto para modificar a sua velocidade, seja a partir do repouso ou a partir de uma velocidade inicial não-nula. Para um 
objeto de massa $m$ a uma velocidade $v$ a sua energia cinética, em um instante de tempo $t$, é expressa como:

$$
E_{c}(t)=\frac{m v^{2}}{2}
$$

Evidenciando $v$ na Equação 5, obtém-se a velocidade de um corpo com energia cinética $E_{c}$ pela Equação 6:

$$
v=\sqrt{\frac{2 E_{c}}{m}}
$$

\subsection{Energia potencial}

A energia potencial $\left(E_{p}\right)$ é conhecida como energia armazenada. A energia potencial, mediante a realização de trabalho, é transformada em outras formas de energia.

Entre as energias potenciais existentes, como a hidráulica e a nuclear, a energia potencial gravitacional é a mais conhecida e estudada nas escolas de ensino médio. Para um objeto de massa $m$ a uma altura $h$ sob a ação da gravidade $g$ a sua energia potencial gravitacional, em um instante de tempo, é calculada pela Equação 7:

$$
E_{p}=m g h
$$

\subsection{Energia mecânica}

A energia mecânica $\left(E_{m}\right)$ de um sistema é a soma das energias que atuam nesse sistema. A energia mecânica de um objeto que possui energia cinética $\left(E_{c}\right)$ e energia potencial $\left(E_{p}\right)$, em um instante de tempo, é calculada através da Equação 8:

$E_{m}=E_{p}+E_{c}$

\subsection{Lei da conservação da energia}

A energia não se cria nem se destrói, apenas se transfere. Basicamente a lei da conservação da energia se resume a isso. A energia mecânica inicial $\left(E_{m i}\right)$ de um sistema isolado conservativo será igual sua energia mecânica final $\left(E_{m f}\right)$ conforme definido na Equação 9:

$S_{\text {conservativo }} \rightarrow E_{m i}=E_{m f}$

\subsection{Lei da conservação da quantidade de movimento}

A lei da conservação da quantidade de movimento diz que a quantidade de movimento de um sistema, quando a resultante das forças externas for nula, é constante. A quantidade de movimento de um objeto é o produto da massa $(m)$ pela velocidade $(v)$ expresso pela Equação 10:

$$
\sum m_{i} v_{i}=\sum m_{f} v_{f}
$$

\subsection{Movimento harmônico simples}

O movimento harmônico simples (MHS) corresponde a um movimento de oscilação com período $T$ constante. $\mathrm{O}$ pêndulo simples, quando oscila com pequena amplitude, descreve um MHS. O período de um pêndulo simples que sustenta um objeto ligado a um fio de comprimento $l$ com a aceleração da gravidade $g$ é dado pela Equação 11:

$$
T=2 \pi \sqrt{\frac{l}{g}}
$$

\subsection{Colisão elástica entre blocos rígidos}

Suponhamos dois blocos de massas $m_{1}$ e $m_{2}$ com velocidades iniciais $\vec{v}_{1 i}$ e $\vec{v}_{2 f}$ respectivamente (Figuras 5.a e 5.b).

Combinando-se a equação da lei de conservação do movimento (Equação 10) com a equação que descreve a energia cinética (Equação 5) escreve-se:

$\frac{m_{1}\left(\vec{v}_{1 i}\right)^{2}}{2}+\frac{m_{2}\left(\vec{v}_{2 i}\right)^{2}}{2}=\frac{m_{1}\left(\vec{v}_{1 f}\right)^{2}}{2}+\frac{m_{2}\left(\vec{v}_{2 f}\right)^{2}}{2}$

Quando as velocidades dos blocos se processam apenas em uma direção podemos usar apenas os módulos das velocidades. Na simulação tem-se $\vec{v}_{2 i}=0$. Por fim, a partir da Equação 12, mostra-se que:

$\vec{v}_{1 f}=\frac{m_{1}-m_{2}}{m_{1}+m_{2}} \vec{v}_{1 i}$

$\vec{v}_{2 f}=\frac{2 m_{1}}{m_{1}+m_{2}} \vec{v}_{1 i}$

Das Equações 13 e 14 surgem dois casos particulares:

(i) Se $m_{1}=m_{2}$ então $\vec{v}_{1 f}=0$ e $\vec{v}_{2 f}=\vec{v}_{1 i}$. O bloco 1 para e transfere toda a sua velocidade para o bloco 2 .

(ii) Se $m_{2}>>m_{1}$ então $\vec{v}_{1 f}=-\vec{v}_{1 i}$ e $\vec{v}_{2 f}=0$. O bloco 2 continua parado sem velocidade e o bloco 1 continua com velocidade de mesmo módulo e sentido, porém em direção contrária a velocidade inicial. 
$\vec{v}_{1 i}$ é diferente de zero e está na direção do bloco 2 .

$$
\vec{v}_{2 \imath}=0
$$$$
m 1=m 2
$$

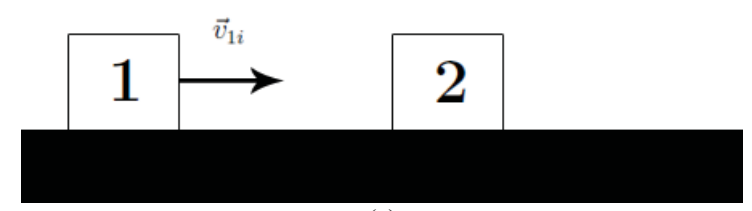

(a)

$$
\begin{aligned}
& \vec{v}_{1 f}=0 \\
& \vec{v}_{2 f}=\vec{v}_{1 i} \\
& m 1=m 2
\end{aligned}
$$

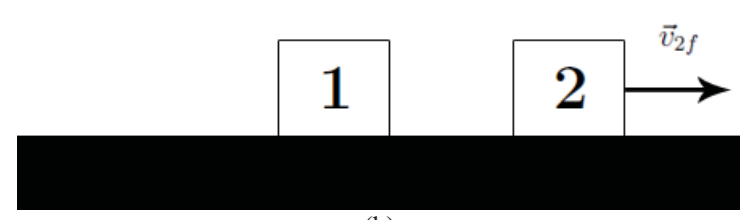

(b)

Figura 5: Colisão elástica entre blocos rígidos de mesma massa. Diagrama de forças antes da colisão (a) e apos a colisão (b).

\subsection{Lançamento vertical para cima e queda livre}

Considere que uma esfera possua massa $m$ e seja inicialmente lançada para cima a partir do solo com velocidade $v$ sobre a ação da gravidade $g$ (Figuras 6.a e 6.b). Pode-se calcular sua energia cinética no instante de tempo igual a zero utilizando a Equação 5. No instante inicial, pode-se afirmar que a energia mecânica do sistema será igual a energia cinética, pois a altura e consequentemente a energia potencial serão iguais a zero (Equação 7). Assim, a energia mecânica da esfera, que deve se manter constante, será proporcional ao quadrado de sua velocidade inicial e inversamente proporcional a sua massa (Equação 5).

Tomando como referência o nível do solo, durante a subida a quantidade de energia cinética diminui e a potencial gravitacional aumenta. Sabe-se que a velocidade é dada pela variação da posição no espaço em relação ao tempo. Assim, a cada instante de tempo e consequentemente a cada variação da altura da esfera, sabe-se o valor da energia potencial gravitacional (Equação 7). Ainda, a energia mecânica do sistema é uma constante, dessa for- ma, sendo $E_{m}$ uma constante e $E_{p}$ conhecida, calcula-se o valor da energia cinética utilizando a Equação 8. A nova velocidade é obtida a partir da Equação 6.

$$
\begin{gathered}
E_{c}=\frac{m v^{2}}{2} \\
E_{p}=0 \\
E_{m}=E_{c} \\
v=\text { máxima } \\
h=0
\end{gathered}
$$
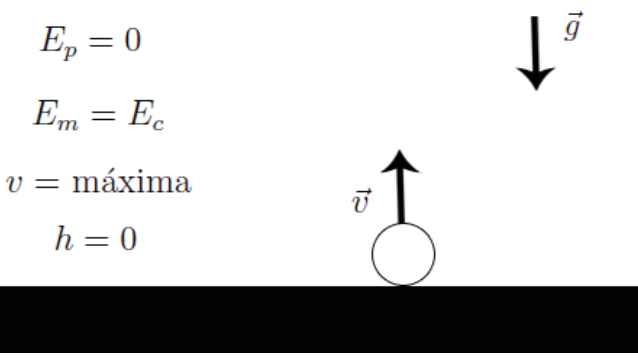

(a)

$$
\begin{gathered}
E_{p}=m h g \\
E_{c}=E_{m}-E_{c} \\
v=\sqrt{\frac{2 E_{c}}{m}}
\end{gathered}
$$
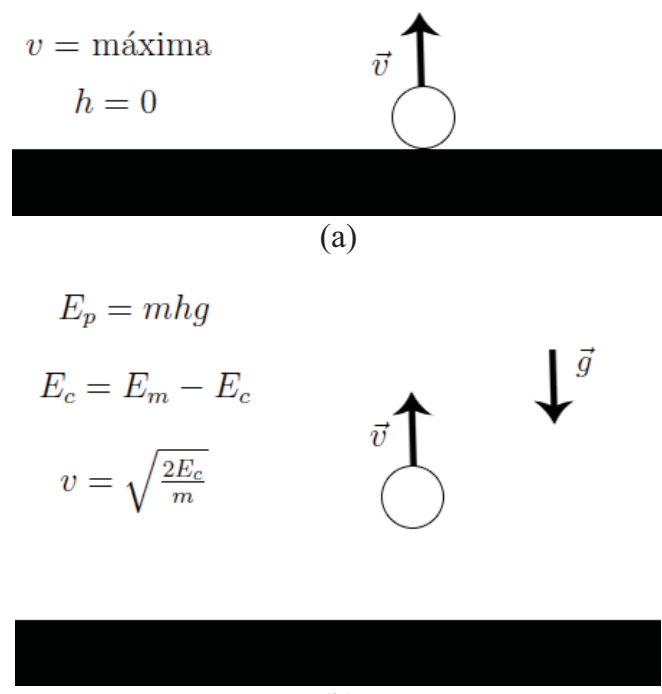

(b)

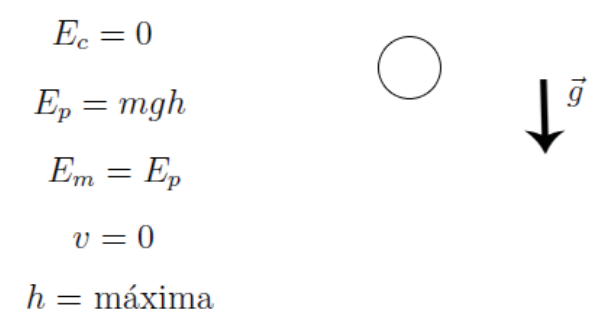

(c)

$$
\begin{gathered}
E_{p}=m h g \\
E_{c}=E_{m}-E_{c} \\
v=\sqrt{\frac{2 E_{c}}{m}}
\end{gathered}
$$
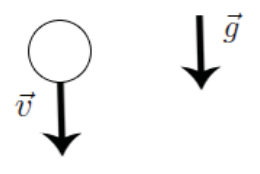

(d)

Figura 6: Lançamento vertical para cima e queda livre. 
Percebe-se que no lançamento vertical para cima o movimento da esfera é uniformemente retardado, pois sua velocidade decresce à medida que varia sua posição. Quando toda a energia cinética é convertida em energia potencial a esfera chega a sua altura máxima. Toda a energia do sistema está armazenada em forma de energia potencial. Nesse momento acontece a inversão de movimento (Figuras 6.c e 6.d) e a esfera é acelerada pela gravidade local.

$\mathrm{Na}$ queda livre o movimento da esfera é uniformemente acelerado pela gravidade $g$. O módulo da velocidade final da esfera ao atingir o solo será igual a velocidade de lançamento, pois, nesse momento, a $E_{p}$ será nula e novamente a $E_{c}$ será igual a $E_{m}$.

\subsection{Descida de um bloco apoiado sobre um plano inclinado}

As forças que atuam sobre um bloco de massa $m$ apoiado sobre um plano inclinado de ângulo $\theta$ em relação à horizontal e sob a ação da gravidade $g$ são: força peso $(\vec{P})$ e força normal $(\vec{N})$. Elas são também conhecidas como: força de atração gravitacional e força de reação ao contato do bloco com a superfície de apoio, respectivamente (Figura 7).

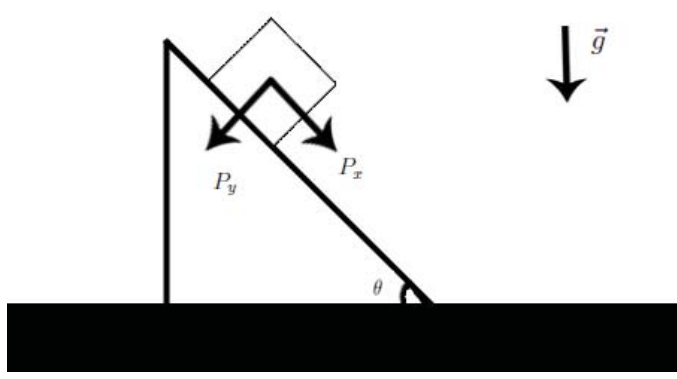

Figura 7: Descida de um bloco apoiado sobre plano inclinado e sob a ação da gravidade.

É costumeiro, para simplificar a análise matemática desse tipo de problema, decompor as forças que atuam sobre o bloco em duas direções. A força $P_{x}$ é paralela ao plano inclinado e $P_{y}$ é perpendicular ao plano inclinado. A primeira é responsável pela descida do bloco enquanto a última é equilibrada pela força normal do plano. Sabese, utilizando geometria triangular e a partir do ângulo $\theta$ da rampa, que essas forças decompostas podem ser calculadas pelas Equações 15 e 16:

$$
\begin{aligned}
& P_{x}=P \sin \theta \\
& P_{y}=P \cos \theta
\end{aligned}
$$

Ainda, a partir da Equação 15, tem-se que:

$$
\begin{aligned}
& P \sin \theta=m a \\
& m g \sin \theta=m a \\
& a=g \sin \theta
\end{aligned}
$$

Como não existe movimento em outras direções temse que:

$$
\begin{aligned}
& N=P_{y} \\
& N=m g \cos \theta
\end{aligned}
$$

\subsection{Pêndulo simples}

Um pêndulo simples é um sistema ideal que consiste de um objeto suspenso por um fio inextensível e leve (Figura 8). Calcula-se o período de um pêndulo simples utilizando a Equação 11 quando a amplitude angular do mesmo é menor que quinze graus.
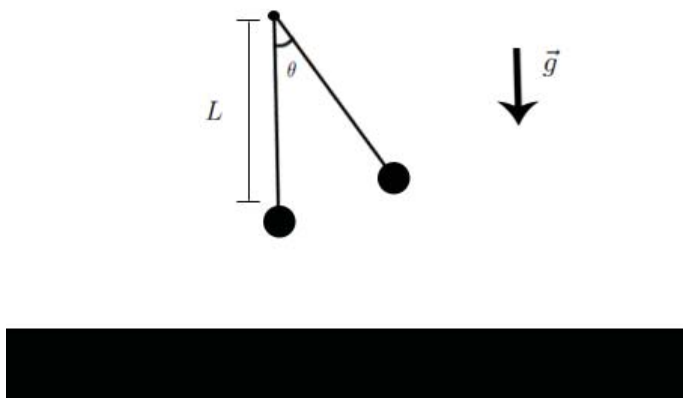

Figura 8: Pêndulo simples\}

Quando afastado de sua posição de equilíbrio e solto, o pêndulo oscilará em um plano vertical sobre a ação da gravidade. Quando o pêndulo oscila em pequenas amplitudes $(\theta<15)$ ele descreve um movimento harmônico simples. Assim, para um pêndulo com um fio de comprimento $l$ sobre a ação da gravidade $g$ e amplitude $\theta<15$ sabe-se que o período $T$ pode ser calculado através da Equação 11. Nessas condições, conclui-se que o período de oscilação independe da amplitude e da massa do objeto, sendo diretamente proporcional à raiz quadrada do comprimento e inversamente proporcional à raiz quadrada da aceleração da gravidade local.

\section{Sistema Proposto}

A ferramenta de apoio ao ensino de física utilizando RA (FAEFRA) foi desenvolvida na linguagem $\mathrm{C}++$ com o auxílio do framework SudaRA apresentado anterior- 
mente. A biblioteca OpenGL (Open Graphics Library) foi utilizada na geração dos objetos da aplicação.

Para que não houvesse a necessidade de utilização de teclado ou mouse durante a execução das simulações, toda a interação necessária (alteração de massa dos objetos, velocidade inicial etc.) é feita através da manipulação de marcadores especiais inseridos na cena. Optou-se por utilizar o ângulo de rotação destes marcadores, para que a posição dos mesmos em relação à câmera fosse alterada o mínimo possível.

A FAEFRA é composta por quatro simulações de eventos físicos: colisão elástica entre blocos rígidos, lançamento vertical para cima e queda livre, descida de um bloco apoiado sobre um plano inclinado e sob a ação da gravidade e o pêndulo simples. Os quatro sistemas desenvolvidos serão apresentados a seguir.

\subsection{Colisão elástica entre blocos rígidos}

A colisão elástica entre dois blocos rígidos permite verificar a conservação do movimento e a conservação de energia mecânica em um sistema isolado. Na simulação proposta desconsidera-se o fato de que uma colisão pode resultar a excitação de um átomo, molécula ou núcleo. Simula-se somente a colisão elástica, quando toda energia cinética do sistema é conservada.

O sistema criado é composto por dois blocos posicionados um de frente para o outro. Durante a simulação é atribuído a um dos blocos uma velocidade inicial na direção do bloco oposto. Essa velocidade permite que o bloco se desloque e atinja o bloco que estava parado.

A inserção do marcador (Figura 9.a) na cena possibilita que ambos os blocos sejam visualizados (Figura 9.b).

O ângulo de rotação do marcador sobre a mesa e em relação a câmera de vídeo determina a massa do bloco que não receberá velocidade inicial. Ao rotacionar o marcador é possível modificar a massa do bloco para $15 \mathrm{~kg}, 3 \mathrm{~kg}$ ou $5000 \mathrm{~kg}$. Esses valores para a massa do bloco possibilitam simular os diferentes casos possíveis na colisão de blocos rígidos. As Figuras 10 e 11 fazem um comparativo entre a rotação do marcador e a massa do bloco desenhado.

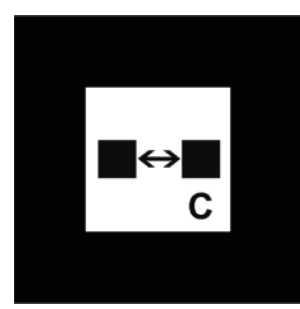

(a)

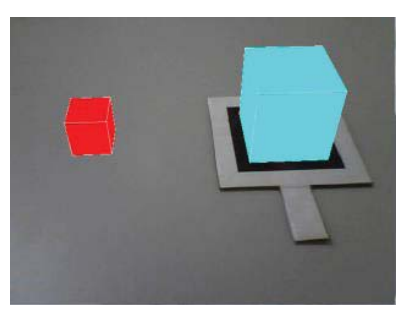

(b)
Figura 9: Blocos criados com a inserção de um marcador. Marca- dor que desenha os blocos na cena (a) e visualização dos blocos quando o marcador é inserido (b).

Outro marcador (Figura 12.a) define a velocidade inicial de um dos blocos. Sendo $\theta$ o ângulo de rotação do marcador em relação a câmera, calcula-se a velocidade através da Equação 22.

$v_{i}=\frac{\theta}{15}$

Determina-se a velocidade dessa forma, pois ela garante uma faixa satisfatória de possíveis velocidades iniciais. Sabe-se que $0<\theta<360$, assim a velocidade máxima inicial será de $24 \mathrm{~m} / \mathrm{s}$ e a mínima estipulada é $1 \mathrm{~m} / \mathrm{s}$.

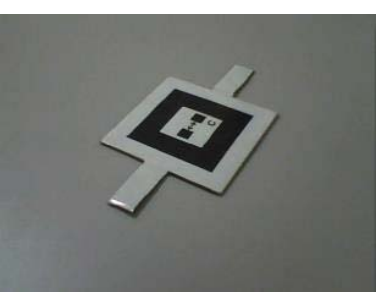

(a)

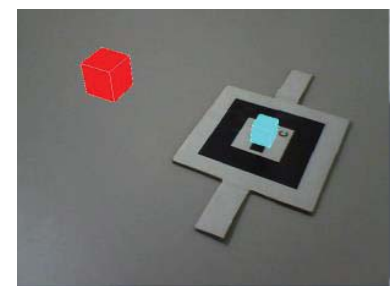

(b)
Figura 10: Ângulo de rotação do marcador para que o bloco tenha massa igual a $3 \mathrm{~kg}$ em (a) e projeção da massa em (b).

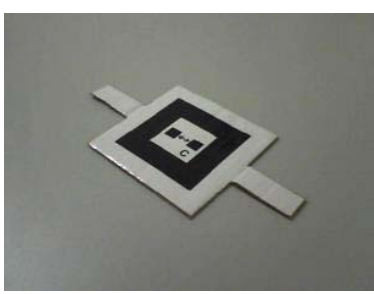

(a)

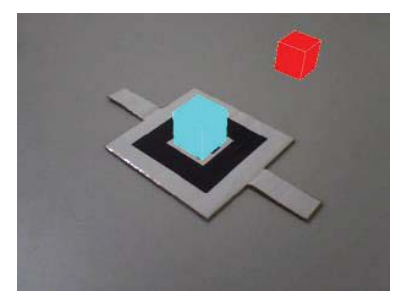

(b)
Figura 11: Ângulo de rotação do marcador para que o bloco tenha massa igual a 15k em (a) e projeção da massa em (b).

Por fim, a inserção do marcador da Figura 12.b na cena inicia a simulação. Quando esse marcador entra em cena a velocidade definida pelo ângulo de rotação do marcador representado na Figura 12.a é atribuída a um dos blocos.

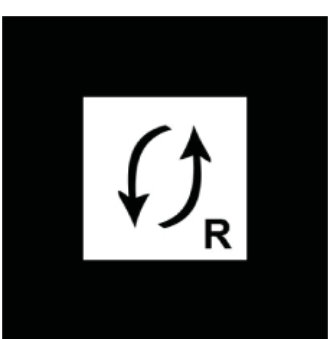

(a)

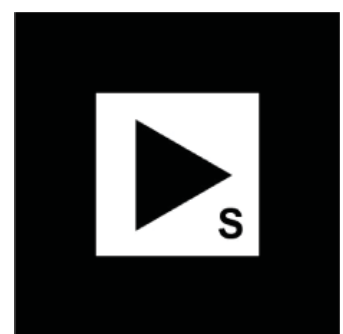

(b)
Figura 12: Marcadores utilizados na simulação. Em (a) marcador que define a velocidade inicial de um dos blocos e em (b) marcador responsável por iniciar a simulação. 
$\mathrm{Na}$ simulação, o bloco que adquirir a velocidade inicial descreve um movimento uniforme com velocidade na direção e sentido do outro bloco. A posição do bloco é calculada utilizando a Equação 2. Sabendo a posição do bloco é possível calcular o instante correto da colisão e é nesse momento que se calcula a velocidade dos blocos após a colisão de acordo com as Equações 13 e 14. Após uma simulação, os cubos são desenhados novamente em suas posições iniciais para que uma nova simulação possa ser feita.

Durante a simulação são impressos os valores da massa e da velocidade de ambos os blocos. Além disso, o valor da velocidade inicial que será atribuída aos blocos é disponibilizado na tela da aplicação.

\subsection{Lançamento vertical para cima e queda livre}

A simulação do lançamento vertical para cima e a queda livre permitem observar o movimento uniformemente variado e a conservação da energia mecânica em um sistema isolado.

No sistema criado, uma esfera inicialmente no solo é arremessada para cima sob a ação da gravidade. Após o lançamento, a esfera atinge a altura máxima de acordo com a velocidade inicial determinada e com a gravidade do local. No instante em que alcança a altura máxima a esfera começa a cair com aceleração constante até atingir o solo.

O marcador da Figura 13.a ao ser inserido na cena possibilita que a esfera seja visualizada no monitor. A imagem da Figura 13.b é o resultado que se obtém com a presença desse marcador no vídeo captado pela câmera.

Ao rotacionar o marcador é possível alterar a massa da esfera para $10 \mathrm{~kg}, 20 \mathrm{~kg}$ ou $30 \mathrm{~kg}$. As Figuras 14.a e 14.b exemplificam o ângulo de rotação do marcador e a massa da esfera gerada na cena.

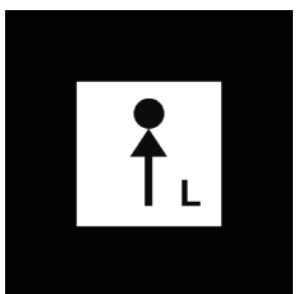

(a)

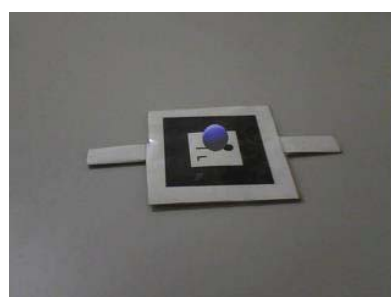

(b)
Figura 13: Esfera criada com a inserção de um marcador (a) e ângulo de rotação do marcador para que o bloco tenha massa de $3 \mathrm{~kg}$ (b).

O marcador da Figura 15.a é responsável por determinar a energia cinética de lançamento. Ignorando a resistência do ar, objetos lançados com a mesma velocidade, mesmo que possuam massas diferentes, descreverão movimentos iguais. Desta forma, optou-se por utilizar o marcador para determinar a energia cinética de lançamento ao invés da velocidade inicial de lançamento. Rotacionando o marcador é possível determinar essa energia para $10.000 \mathrm{~J}, 15.000 \mathrm{~J}$ e $25.000 \mathrm{~J}$.

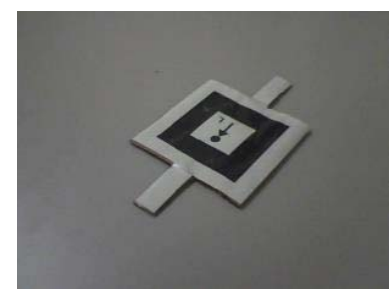

(a)

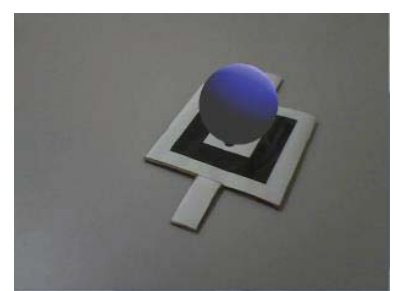

(b)
Figura 14: Ângulo de rotação do marcador (a) para que o bloco tenha massa igual a $30 \mathrm{~kg}(\mathrm{~b})$.

O marcador da Figura 15.b, ao ser inserido na cena, calcula a velocidade inicial de lançamento de acordo com a energia cinética e a massa definidas pelos marcadores citados anteriormente e inicia o lançamento da esfera.

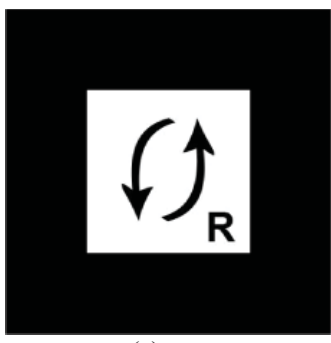

(a)

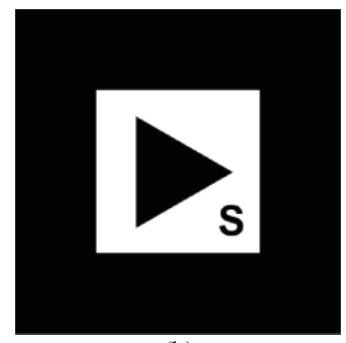

(b)
Figura 15: Marcadores utilizados no lançamento vertical e na queda livre. Em (a) a energia cinética de lançamento é determinada e em (b) tem-se o marcador que inicia a simulação.

Durante a subida, o objeto descreve um movimento uniformemente desacelerado, pois o sentido da velocidade do objeto e da aceleração da gravidade são iguais, mas os sentidos de ambos são opostos. Na descida, a velocidade do objeto e a aceleração da gravidade possuem sentido e direção iguais e portanto o objeto descreve um movimento uniformemente acelerado.

A cada instante de tempo calcula-se a velocidade da esfera de acordo com a Equação 4, lembrando que a aceleração exerce força contrária ao eixo positivo horizontal definido, então a aceleração é dada por - $g$. A posição no instante $t+1$ da esfera pode ser calculada utilizando a velocidade e a posição do cubo no instante $t$. Sabendo a posição e a velocidade da esfera calcula-se sua energia cinética e sua energia potencial.

Durante a simulação, os valores da energia mecânica da esfera, da altura máxima atingida, da velocidade inicial e da massa da esfera são impressos na tela da aplicação para que o usuário possa acompanhar os cálculos que envolvem o evento físico simulado. 


\subsection{Descida de um bloco sobre um plano inclinado}

A descida de um bloco sobre um plano inclinado permite observar a decomposição de forças, o movimento uniformemente variado e a conservação da energia mecânica em um sistema isolado. O marcador utilizado para desenhar o bloco e o plano pode ser observado na Figura 16.a e sua respectiva representação no sistema na Figura $16 . \mathrm{b}$

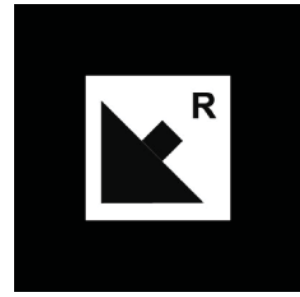

(a)

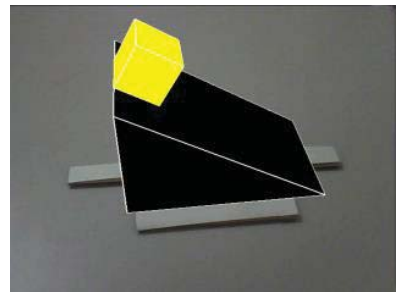

(b)
Figura 16: Em (a) marcador que desenha o bloco e o plano inclinado e em (b) a visualização do sistema.

Um bloco é posicionado no topo de um plano inclinado de ângulo $\theta$ em relação ao plano horizontal. Sob a ação da gravidade esse bloco desliza pelo plano inclinado até atingir o plano horizontal. O sistema implementado simulará essa situação.

Ao ser inserido na cena, o marcador ilustrado na Figura 17.a possibilita que o plano inclinado e o cubo sejam visualizados pelo usuário (Figura 17.b). Esse marcador, quando rotacionado, altera o valor do ângulo $\theta$. Os possíveis valores de $\theta$ são: 30, 45 e 60 graus.

A inserção do marcador da Figura 18.b na cena inicia a descida do cubo pelo plano inclinado. O marcador representado na Figura 18.a é responsável por voltar o cubo para a sua posição inicial, no topo do plano inclinado.

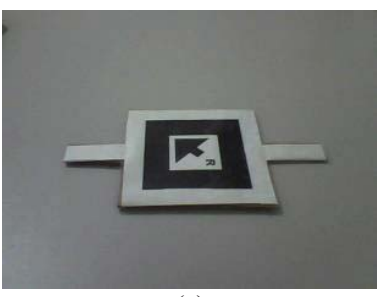

(a)

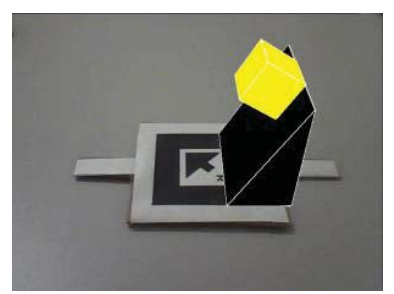

(b)
Figura 17: Ângulo de rotação do marcador para $\theta=60$ graus (a) e sua representação no sistema (b).

O bloco, durante a descida, descreve um movimento acelerado de acordo com a gravidade local e o valor do ângulo $\theta$. A Equação 19 foi utilizada para calcular a aceleração constante com que o bloco desce a rampa. Ainda, a velocidade pode ser calculada através da Equação 4. Com esses dados pode-se simular a descida do bloco calculando sua posição a cada instante de tempo de acordo com a velocidade do bloco no momento e a posição do mesmo no instante de tempo anterior.

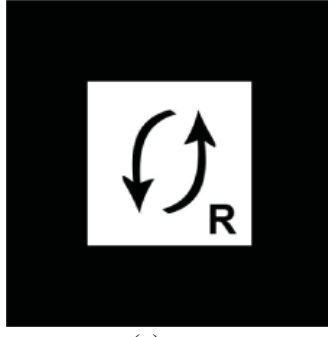

(a)

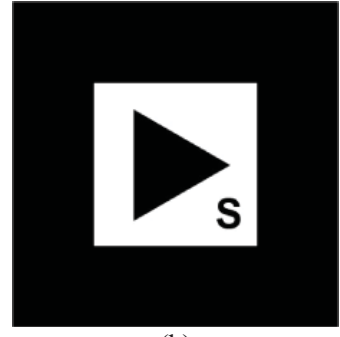

(b)
Figura 18: Marcadores utilizados na simulação da descida de um bloco sobre um plano inclinado. O marcador em (a) posiciona o cubo na rampa e em (b) inicia a descida.

Durante a simulação, os valores da velocidade e aceleração do bloco são impressos no vídeo gerado. Além disso, também aparecem para o usuário o tempo gasto para o bloco descer todo o plano inclinado e o valor do ângulo $\theta$.

\subsection{Pêndulo simples}

O pêndulo simples será utilizado para demonstrar o movimento harmônico simples.

Para a simulação desse sistema físico foram utilizados dois marcadores. A inserção do marcador ilustrado na Figura 19.a na cena possibilita ao usuário visualizar o pêndulo e o seu movimento harmônico simples. O segundo marcador ilustrado na Figura 19.b, ao ser rotacionado, modifica o comprimento do fio. O resultado obtido pela inserção do marcador de inclusão do pêndulo na cena pode ser visto nas Figuras 20.a e 20.b. A alteração do comprimento do fio pode ser observada nas Figuras 21.a e 21.b.

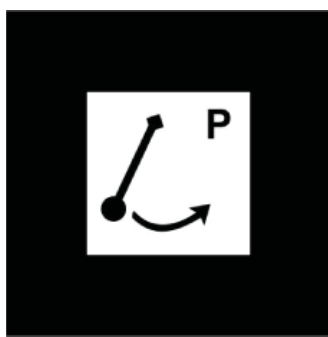

(a)

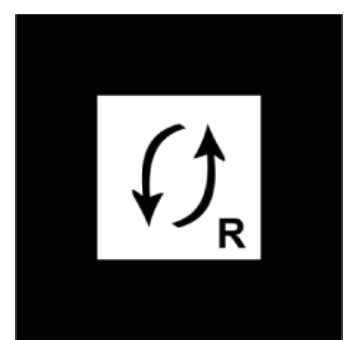

(b)
Figura 19: Marcadores utilizados na simulação do pêndulo simples.

O período do pêndulo só depende da gravidade do local e do comprimento do fio. Assim, optou-se por incluir um marcador que altera esse comprimento, forçando o cálculo do novo período e consequentemente gerando 
simulações diferentes para o mesmo evento físico.

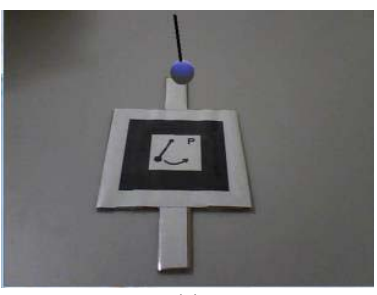

(a)

Figura 20: Pêndulo visualizado na cena.

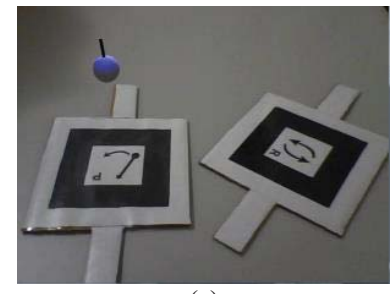

(a)

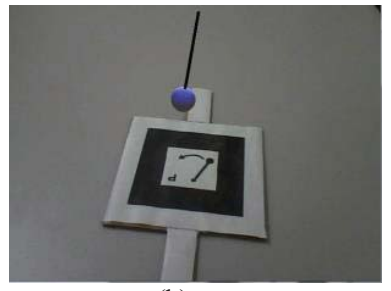

(b)

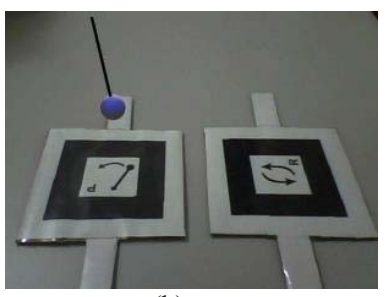

(b)
Figura 21: Modificando o comprimento do fio de acordo com ângulo de rotação do marcador. Em (a) comprimento do fio foi igual a $10 \mathrm{~m}$ e em (b), $30 \mathrm{~m}$.

Durante a simulação foram impressos os valores da gravidade local, do período e do comprimento do fio. Através disso e com o auxílio da Equação 11 o usuário poderá conferir os cálculos que determinam o período de um pêndulo simples.

\section{Resultados}

Nesta seção serão apresentados os resultados obtidos pelo sistema proposto. Estes resultados estão disponíveis na internet em vídeo [16].

\subsection{Colisão elástica entre blocos rígidos}

Com as possibilidades de combinações, três casos de testes foram criados. No primeiro caso o bloco 1 e o bloco 2 possuem massas iguais. Assim como esperado, ao colidir, a energia cinética do bloco 1 foi totalmente transferida para o bloco 2. A velocidade do bloco 2 após a colisão é igual a velocidade do bloco 1 antes da colisão. O bloco 1 fica parado após a colisão (Figuras 22.a e 22.b).

No segundo caso o bloco 2 possui massa muito maior que a do bloco 1 . Como previsto, após a colisão a velocidade do bloco 1 será igual em módulo, porém em direção contrária. O bloco 2 não se mexe durante a colisão (Figuras 23.a e 23.b).

No terceiro caso a massa do bloco 2 é vinte por cento da massa do bloco 1. Após a colisão, ambos os blocos possuem velocidade (Figuras 24.a e 24.b).

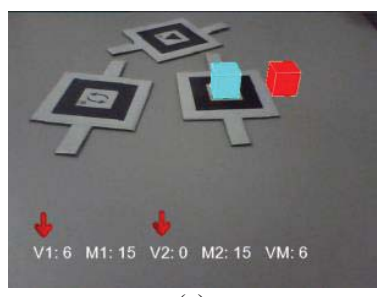

(a)

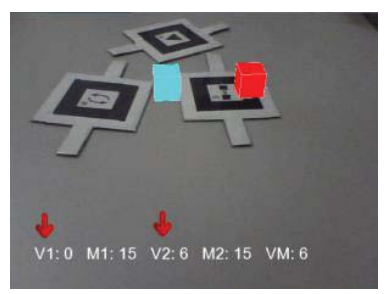

(b)
Figura 22: Colisão elástica entre blocos sólidos de mesma massa. Em (a), simulação antes da colisão e em (b), após.

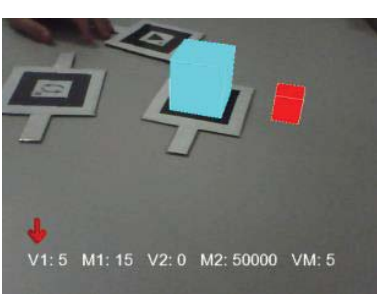

(a)

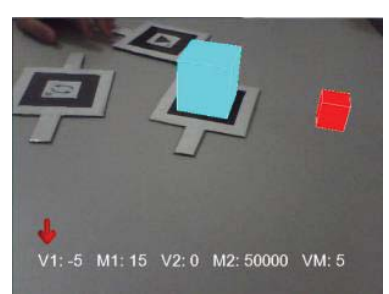

(b)
Figura 23: Colisão elástica entre blocos sólidos de massas diferentes. Sistema antes (a) e depois (b) da colisão.

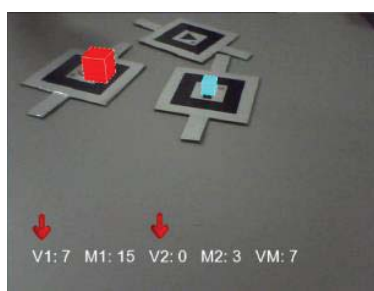

(a)

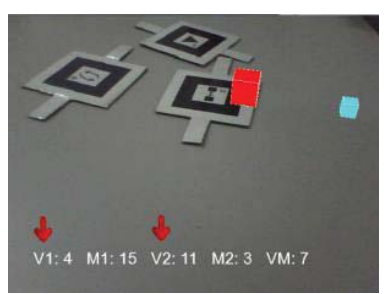

(b)
Figura 24: Colisão elástica entre blocos sólidos de massas diferentes. M1 > M2. Sistema antes (a) e depois (b) da colisão.

Os resultados esperados foram obtidos e os mesmos estão resumidos na Tabela 1. Com a simulação foi possível perceber que a velocidade inicial do bloco 1 e o peso do bloco 2 são determinantes para o resultado final da colisão.

Tabela 1: Resultados obtidos na colisão elástica entre blocos rígidos.
\begin{tabular}{|c|c|c|c|c|c|}
\hline$P_{1}(\mathrm{Kg})$ & $P_{2}(\mathrm{Kg})$ & $V_{1 i}(\mathrm{~m} / \mathrm{s})$ & $V_{2 i}(\mathrm{~m} / \mathrm{s})$ & $V_{1 f}(\mathrm{~m} / \mathrm{s})$ & $V_{2 f}(\mathrm{~m} / \mathrm{s})$ \\
\hline 15 & 15 & 6 & 0 & 0 & 6 \\
\hline 15 & 5000 & 5 & 0 & -5 & 0 \\
\hline 15 & 3 & 7 & 0 & 4 & 11 \\
\hline
\end{tabular}

\subsection{Lançamento vertical para cima e queda livre}

Os testes realizados envolveram esferas de massas diferentes. Para cada teste aplicou-se uma energia cinética inicial de lançamento diferente.

Na primeira simulação e no instante $t=0 \mathrm{o}$ valor da energia cinética foi $10.000 \mathrm{~J}$. A velocidade de lançamento 
da esfera dependerá de seu peso sendo calculada nos testes através da Equação 6. Sabe-se que essa velocidade será inversamente proporcional a raiz quadrada de sua massa. Os resultados para essa simulação podem ser observados nas Figuras 25.a e 25.b. Utilizando-se do mesmo princípio explicado anteriormente, será calcula a velocidade de lançamento quando a energia cinética no instante $t=0$ for igual a $\mathrm{E}_{\mathrm{c}}=15.000 \mathrm{~J}$. Os resultados desta segunda simulação estão expostos nas Figuras 26.a e 26.b.

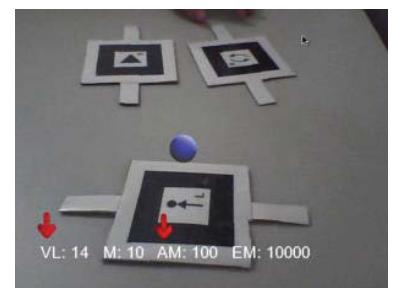

(a)

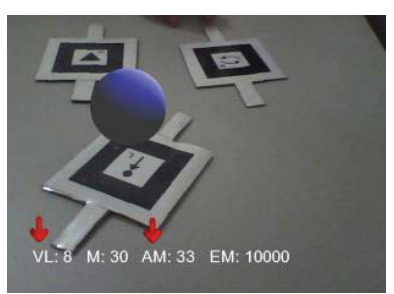

(b)
Figura 25: Lançamento vertical para cima e queda livre $\mathrm{E}_{\mathrm{m}}=$ 10.000J e $\mathrm{M}=10$ (a) e $\mathrm{M}=30$ (b).

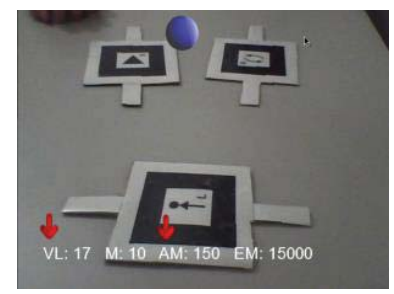

(a)

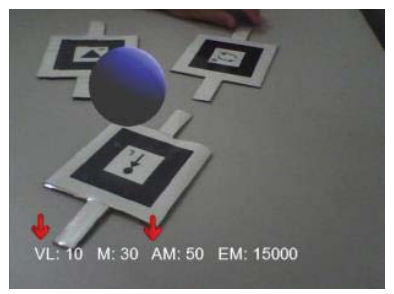

(b)
Figura 26: Lançamento vertical para cima e queda livre $\mathrm{E}_{\mathrm{m}}=$ $15.000 \mathrm{~J}$ e $\mathrm{M}=10$ (a) e $\mathrm{M}=30$ (b).

Os resultados esperados foram obtidos e os mesmos estão organizados na Tabela 2. Durante as simulações foi possível perceber o movimento uniformemente variado da esfera.

Tabela 2: Resultados obtidos no lançamento vertical e queda livre.

\begin{tabular}{|c|c|c|c|}
\hline $\begin{array}{c}\text { Peso } \\
(\mathrm{Kg})\end{array}$ & $\begin{array}{c}\text { Energia } \\
\text { Mecânica }(\mathrm{J})\end{array}$ & $\begin{array}{c}\text { Velocidade de } \\
\text { lançamento }(\mathrm{m} / \mathrm{s})\end{array}$ & $\begin{array}{c}\text { Altura Máxima } \\
(\mathrm{m})\end{array}$ \\
\hline 10 & 10.000 & 14 & 100 \\
\hline 20 & 10.000 & 10 & 50 \\
\hline 30 & 10.000 & 8 & 33 \\
\hline 10 & 15.000 & 14 & 150 \\
\hline 20 & 15.000 & 12 & 75 \\
\hline 30 & 15.000 & 10 & 50 \\
\hline
\end{tabular}

\subsection{Descida de um bloco apoiado sobre um plano inclinado}

Sabe-se que a aceleração do cubo durante a descida é determinada somente pela gravidade do local e o ângulo do plano inclinado com o plano horizontal. Nos casos de teste a gravidade local foi mantida constante enquanto o ângulo entre os planos foi alterado.
Nas Figuras 27, 28 e 29 tem-se os resultados da simulação proposta. Nelas, pode-se observar os valores da aceleração e da velocidade durante a descida do bloco.

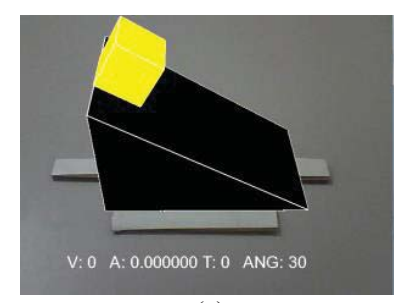

(a)

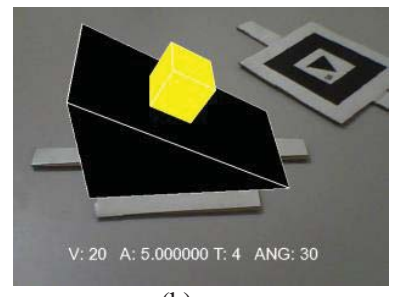

(b)
Figura 27: Estado do sistema $\operatorname{com} \theta=30$ no início (a) e durante (b) a simulação.

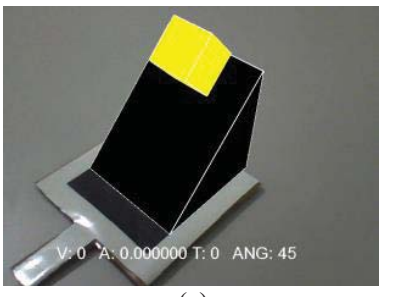

(a)

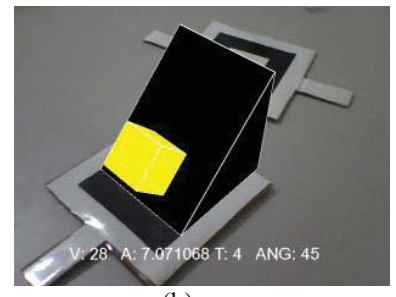

(b)
Figura 28: Estado do sistema com $\theta=45$ no início (a) e durante (b) a simulação.

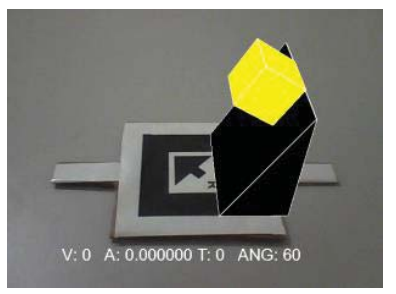

(a)

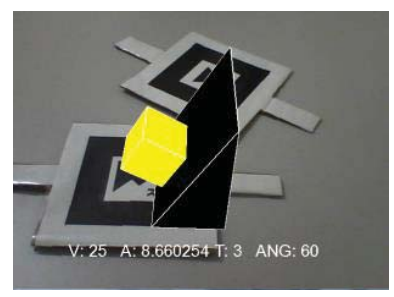

(b)
Figura 29: Estado do sistema com $\theta=60$ no início (a) e durante (b) a simulação.

A Tabela 3 mostra os resultados obtidos para a aceleração dos blocos quando o ângulo entre o plano horizontal e o plano inclinado varia entre 15,30 e 45 graus.

Tabela 3: Resultados obtidos na descida de um bloco apoiado sobre um plano inclinado e sob a ação da gravidade local de $10 \mathrm{~m} / \mathrm{s}^{2}$.

\begin{tabular}{|c|c|}
\hline Ângulo $(\theta)$ em graus & Aceleração $\left(m / s^{2}\right)$ \\
\hline 30 & 5 \\
\hline 45 & 7,06 \\
\hline 60 & 8,66 \\
\hline
\end{tabular}

\subsection{Pêndulo simples}

As simulações do pêndulo simples mantiveram a gravidade e a amplitude $\theta$ constantes. Durante os testes somente o comprimento do fio foi alterado.

O período obtido para cada comprimento de fio estipulado correspondeu ao esperado pela Equação 11. Os resultados obtidos através da simulação de pêndulo sim- 
ples estão expostos nas Figuras 30.a e 30.b e estão simplificados na Tabela 4. Durante a simulação foi possível acompanhar a trajetória do movimento harmônico simples realizada pelo pêndulo.

Tabela 4: Resultados obtidos na simulação de um pêndulo simples sob a ação da gravidade local de $10 \mathrm{~m} / \mathrm{s}^{2}$.

\begin{tabular}{|c|c|}
\hline Comprimento do Fio $(\mathrm{m})$ & Período do pêndulo $(\mathrm{s})$ \\
\hline 30 & 10 \\
\hline 20 & 8 \\
\hline
\end{tabular}

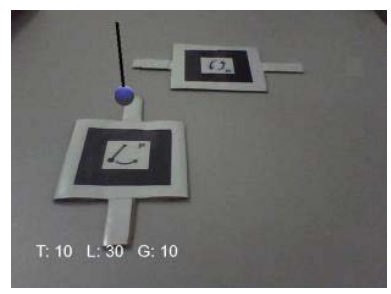

(a)

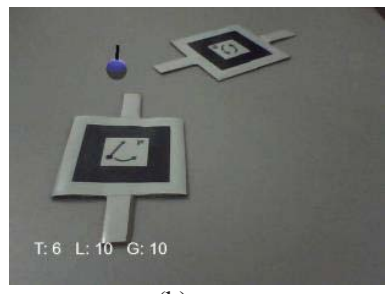

(b)
Figura 30: Pêndulo simples. $\theta<15$ e L $=10$ (a) e $\mathrm{L}=30$ (b).

\section{Conclusão}

Este trabalho apresenta uma ferramenta de apoio ao ensino de física utilizando RA.

Todos as classes necessárias para o funcionamento dos módulos propostos foram implementadas sem o auxílio de bibliotecas auxiliares.

O modelo computacional elaborado foi dividido em módulos e cada módulo simulou um evento físico diferente. Os conceitos físicos necessários para desenvolver as aplicações foram abordados.

Foram realizados experimentos para a análise do sistema implementado. Para cada módulo, verificou-se se os conceitos físicos foram devidamente implementados e se os mesmos poderiam ser explicados utilizando a ferramenta.

Na colisão elástica entre blocos pretendia-se exemplificar a lei de conservação do movimento. Com a análise de três diferentes tipos de colisões foi possível obter resultados de acordo com o esperado. Utilizando-se de esferas e velocidades de lançamento diferentes foi possível simular e comprovar as transformações de energia através do lançamento vertical para cima e a queda livre. O movimento uniformemente acelerado e a decomposição de forças foram devidamente exemplificados na descida de um bloco sobre um plano inclinado. Os diferentes ângulos entre o plano inclinado e o plano horizontal comprovaram que apesar da velocidade final ser igual entre as distintas simulações, a aceleração e o tempo de descida foram diferentes. Por fim, com o pêndulo simples, simu- lou-se o movimento harmônico simples obtendo, para amplitudes pequenas, o período do pêndulo para diferentes tamanhos do fio.

Os primeiros resultados experimentais serão avaliados no primeiro semestre de 2013 quando o protótipo será disponibilizado ao Centro de Ciências da Universidade Federal de Juiz de Fora. Este Centro é um órgão de caráter multidisciplinar que desenvolve e apóia atividades relacionadas à Educação Científica, investigando questões relacionadas à inovação dessa modalidade de ensino. $\mathrm{O}$ órgão desenvolve, ainda, atividades relacionadas à Educação Científica não formal e à divulgação das Ciências e da Cultura Científica, para com isso despertar o interesse pelas Ciências na população e nos estudantes. O espaço oferece atividades, cursos e programas de formação continuada para professores da Educação Básica, Feira de Ciências e realiza exposições de materiais pertencentes ao seu próprio acervo ou ao de outros Centros de Ciências.

Para trabalhos futuros pretende-se aumentar o grau de liberdade do usuário em determinar as variáveis da simulação. Abordar novos eventos físicos e integrá-los entre si é uma prioridade para futuras implementações. Assim, um bloco que desce por um plano inclinado colide com um outro bloco localizado no plano horizontal. Por fim, o atrito e a resistência do ar poderão ser implementados como funcionalidades opcionais de cada evento físico simulado.

\section{Referências}

[1] H. Kato, M. Billinghurst. Marker tracking and hmd calibration for a video-based augmented reality conferencing system. Proceedings of the 2nd IEEE and ACM International Workshop on Augmented Reality (IWAR 99), 1999.

[2] R. Azuma. A survey of augmented reality. Presence-Teleoperators and Virtual Environments, 6(4): 10-20, 1995.

[3] R. Azuma, Y, Baillot, R. Behringer, S. Feiner, S. Julier, B, MacIntyre. Recent advances in augmented reality. Computer Graphics and Applications, 21(6): 34-47, 2001.

[4] M. Fjeld, P. Juchli, B. Voegtli. Chemistry education: A tangible interaction approach. Humancomputer interaction: INTERACT'03; IFIP TC13 International Conference on HumanComputer Interaction, 287-294, 2003.

[5] H. Kaufmann, D. Schmalstieg. Mathematics and geometry education with collaborative augmented reality. Computers \& Graphics, 27(3): 339- 
345, 2003.

[6] H. Kaufmann, B. Meyer. Simulating educational physical experiments in augmented reality. $A C M$ SIGGRAPH ASIA 2008 educators programme, 1-8, 2008.

[7] C. Kirner, E. Zorzal. Aplicações educacionais em ambientes colaborativos com realidade aumentada. Anais do Simpósio Brasileiro de Informática na Educação, 1(1): 114-124, 2005.

[8] R. Tori, C. Kirner, R. Siscoutto. Fundamentos e tecnologia de realidade virtual e aumentada. Editora SBC, São Paulo, 2006.

[9] F. Liarokapis, P. Petridis, P. Lister, M. White. Multimedia augmented reality interface for elearning (marie). World Transactions on Engineering and Technology Education, 1(2): 173-176, 2002.

[10] P. Maier, G. Klinker, M. Tonnis. Augmented reality for teaching spatial relations. Conference of the International Journal of Arts \& Sciences. Citeseer, 2009.

[11] D. Schmalstieg, A. Fuhrmann, Z. Szalavari, M. Gervautz. Studierstube - An environment for collaboration in augmented reality. Virtual Reality, 3(1): 37-48, 1998.

[12] T. Sielhorst, T. Obst, R. Burgkart, R. Riener, N. Navab. An augmented reality delivery simulator for medical training. International Workshop on
Augmented Environments for Medical ImagingMICCAI Satellite Workshop, 141. 2004.

[13] C. Henrique. Suporte ao desenvolvimento de aplicações em realidade aumentada. VII Workshop de Realidade Virtual e Aumentada. http://sudara.sourceforge.net, Mar. 2012.

[14] Z. Zhou, A. Cheok, T. Chan, J. Pan, Y. Li. Interactive entertainment systems using tangible cubes. Proceedings of IE2004, Australian Workshop on Interactive Entertainment, 19-22, 2004.

[15] E. Zorzal, C. Kirner, A. Cardoso. E, Lamounier, M. Oliveira, L. Silva. Ambientes educacionais colaborativos com realidade aumentada. RENOTE, 6(1), 2010.

[16] R. Silva. Ferramenta de Apoio ao Ensino de Física utilizando Realidade Aumentada. Disponível em <https://www.youtube.com/watch?v= LWXOa6QQO4c>. Acesso em: 04 Set. 2012. 